\title{
LA DOCTRINA TOMISTA CLASICA SOBRE EL SIGNO: DOMINGO DE SOTO, FRANCISCO DE ARAÚJO Y JUAN DE SANTO TOMÁS
}

\author{
MAURICIO BEUCHOT* \\ Universidad Naciona? \\ Autónoma de Mérico
}

\section{Introducción}

La tradición tomista dedicaba un apartado muy importante al estudio del signo. Puede llamársele tratamiento "semiótico", ya que corresponde a lo que actualmente se considera como tal, i.e. el estudio general del signo en cuanto tal. ${ }^{1}$ Este tratado del signo se inscribía en una perspectiva más amplia, la de la filosofía. Dentro de la filosofía, era ubicado en el ámbito de la lógica, pero en realidad llevaba no solamente la visión lógica, sino también ontológica, psicológica y gnoseológica. Además, a pesar de que el signo era estudiado en cuanto tal, la orientación principal se dirigía hacia el signo lingüístico. ${ }^{2}$ De esta manera, resultaba una semiótica lógica. ${ }^{3}$ Para nuestra consideración de la teoría del signo en la tradición tomista, atenderemos a tres autores del llamado "renacimiento tomista ibérico", de los siglos xvi-xvII, a saber, Domingo de Soto (1495-1560), Francisco de Araújo (15801664) y Juan Poinsot o Juan de Santo Tomás (1589-1644). En una primera aproximación, podemos entresacar los siguientes elementos comunes a nuestros tres autores. La definición del signo - tomado en general, en cuanto abarca a todas las demás clases de signo- es: aquello que representa algo distinto de sí mismo a la facultad cognoscitiva. Se dividía entonces en diversas clases de signos, con diversas divisio-

- Deseo expresar mi agradecimiento al profesor Mark do Bretton Platts.

1 Cfr. M. Beuchot, Elementos de Semiótica, México: UNAM, 1979, p. 9.

2 Cfr. Idem., "La filosofía del lengraje de Pedro Hispano", en Revista de Filosofía, Mérico 12 (1979), p. 217.

3 Cfr. Idem. La filosofía del lenguaje en la Edad Media, México: UNAM, Introducción. En prensa. 
nes, de acuerdo a diversos fundamentos. Las principales $d i$ visiones eran: (a) según la relación a la facultad cognoscitiva, la división era en formal e instrumental; (b) según la relación a lo significado o designado, la división era en natural y artificial, subdividiéndose este último en convencional y consuetudinario. Cada uno de ellos tenía su propia definición, $y$, a veces, sus propias divisiones. Veamos los problemas que surgían en torno a ellos.

\section{Lógica del signo}

La lógica del signo abarca especialmente el estudio de su definición y su división, pues es un instrumento lógico y, en cuanto ciencia, reflexiona sobre sus instrumentos. En esto el maestro fue Soto, seguido por Araújo y Poinsot.

Definición del signo

El punto de partida del tratamiento sobre el signo en la escolástica es la célebre definición de San Agustín: "El signo es la cosa que, además de la especie que entrega a los sentidos, hace llegar al conocimiento de algo distinto." Esta definición pasa a los escolásticos a través del libro de texto por excelencia para la teología, la obra de Pedro Lombardo: Libri quatour Sententiarum. Santo Tomás la recoge en su comentario a esa obra. ${ }^{\circ}$ Pero tal definición es criticada por incompleta. El mismo Santo Tomás, en ese lugar, dice que sólo abarca a los signos sensibles, y deja de lado muchas cosas que, aun siendo solamente inteligibles, pueden ser signos (p. ej. los conceptos). Domingo de Soto precisa la crítica de Santo Tomás, diciendo que tal definición sólo se aplica a los signos instrumentales, dejando fuera a los demás tipos de signo. ${ }^{7}$ Francisco de Araújo se adhiere a la apreciación de

4 "Signum est res quae praeter speciem quam ingerit sensibus, aliquid aliud facit in cognitionem venire" (S. Agustín, De doctrina christiana, lib. 2, c. 1).

5 Cfr. P. Lombardo, Libri IV Sententiarum, lib. 4, d. 1; ed. Quaracchi, 1916.

- Cfr. Sto. Tomás, In IV Setentiarum, d. 1, q. 1, a. 1, quaestiunc. 2.

7 Cfr. D. de Soto, Summulae, Salmanticae: D. a Portonariis, 1575, lib. 1, c. 2 , lecc. 1, p. 4 r. 
Soto $;^{8}$ y lo mismo hace Juan Poinsot (más conocido como Juan de Santo Tomás). ${ }^{9}$ Con lo cual se percatan de que no se puede tomar como definición del signo en cuanto tal.

Hay que elaborar, pues, una definición del signo en general. Encontramos en Soto esta definición: "Aquello que representa algo distinto de sí mismo a la facultad cognoscitiva." ${ }^{10}$ Esta definición es a la vez más precisa y amplia, abarcando de una manera general a todo signo. ${ }^{11}$ Puede tomarse a Soto como el iniciador de la vertiente que seguirán Araújo y Poinsot. ${ }^{12}$

En cuanto a la definición del signo, Soto la trata como el concepto del significar. El significar puede tomarse de dos maneras, (i) en general, en cuanto conviene tanto a las voces como a los signos que no son términos, y (ii) en especial, en cuanto conviene sólo a las voces y términos. Lo toma en general y, así tomado, el significar es representar algo (distinto del signo mismo) a la facultad cognoscitiva. De este modo puede aplicarse a cosas que no son términos, como el tañer de la campana que evoca la lección; y también a cosas que son términos, como la voz "Jesús" que evoea a Cristo.

Vemos que la definición señala tres elementos: facultad cognoscitiva, algo, y representar. La facultad cognoscitiva

8 Cfr. F. de Araújo, Commentariorum in universam Aristotelis Metaphysicam tomus primus, Burgis et Salmanticae: J. B. Varesius, 1617, lib. 3, q. 2, a. 2, dub. 1, praenot. 1.

${ }^{\theta}$ Cfr. J. de Sto. Tomás, Ars logica seu de forma et materia ratiocinandi, pars II, q. 21, a. 1; ed. B. Reiser, Turín: Marietti, 1930, p. 646a.

10 En la obra citada de Soto, lib. 1, c. 2, texto, p. 3v, se formula así: "quod potentiae cognoscitivae aliquid repraesentat"; hemos puesto, porque nos parece más acertada, la reformulación de su compilador Cosme de Lerma: "quod potentiae cognoscitivae aliquid aliud a se repraesentat" (D. de Soto y C. de Lerma, Compendium Sumularum, 5a. ed. por D. Díaz de Cossío, Burgis: Azpilcueta, 1665 , lib. 1 , s. 1 , n. 1 , p. la).

11 Adviértase la semejanza de esta definición con la de Peirce. Cfr. Collected Papers of Charles Sanders Peirce, ed. por Ch. Hartshorne y P. Weiss, Cambridge, Mass.: The Belknap Press of Harvard University, 1965, vol. 2, parágrafo 2.228.

${ }^{12}$ Araújo define: "Signi vero communis ratio ut sic, in eo consistit ut aliud a se potentiae cognoscitivae repraesentet" ( $O$ p. cit., dub. 1 , praen. 2). Y Poinsot: "Id, quod potentiae cognoscitivae aliquid aliud a se repraesentat" (Op. cit., pars I, lib. 1, c. 2, p. 9a y pars II, q. 21, a. 1, p. 646a). 
debe entenderse en su doble aspecto: tanto inteligible como sensible; de esta manera se puede aplicar al caso de los animales, quienes también emplean signos. El representar consiste en hacer conocer; respecto de ello, hay que decir que hay cuatro causas del hacer conocer: objetiva, efectiva, formal e instrumental. Soto lo ilustra con el ejemplo de la imagen del emperador, que es signo en cuanto lo representa. Así, la imagen o pintura del emperador hace conocer objetivamente, por lo cual es la causa objetiva; la vista hace conocer efectivamente, por lo que es la causa efectiva o eficiente; la noticia visiva que se produce por la interrelación de las dos anteriores hace conocer formalmente, por lo cual es la causa formal; y el aspecto de la noticia bajo el cual se recuerda la imagen del emperador hace conocer instrumentalmente, por lo que es la causa instrumental. El algo que se representa es tomado de manera amplia, como todo aquello que de cualquier manera se significa por cualquier tipo de signo. Es el objeto, y hay tres tipos de objeto: uno que es sólo motivo, otro que es sólo terminativo, y otro que es motivo y terminativo al mismo tiempo. El sólo motivo es el que mueve la facultad a formar una noticia, no de él, sino representativa de otra cosa, como lo hace la imagen con respecto a la noticia del emperador. El sólo terminativo es la cosa conocida por la noticia producida por otro objeto distinto (i.e. el sólo motivo), como el emperador con respecto de su misma noticia. El motivo y terminativo es el que mueve la facultad a formar una noticia de sí mismo, como la imagen respecto de su propia noticia.

Pero no hay que identificar significar con representar, uno es más amplio que el otro. Porque representar es hacer presente, y una cosa puede hacerse presente o representarse a sí misma en cuanto objeto al mismo tiempo motivo y terminativo. En cambio, significar es hacerse presente sólo como objeto motivo, pues nada es signo de sí mismo. De aquí que sean diferentes. $Y$ así como hay varios modos de hacer conocer - los cuatro enumerados: de manera efectiva, objetiva, formal e instrumental一, así también hay varios modos de 
representar y de significar. Hay tres modos de representar: de manera objetiva, formal e instrumental. Pero sólo hay dos modos de significar: de manera formal e instrumental. ${ }^{13}$

Poinsot acepta igualmente el conocimiento de la facultad en dos sentidos: intelectual y sensorial, de modo que los animales usan propiamente signos. ${ }^{1 *}$ Señala también las cuatro causas del conocer: eficiente, objetiva, formal e instrumental. Sigue muy de cerca a Soto, y sólo puede decirse que puntualiza mejor sus definiciones. La causa eficiente del conocer es la misma facultad, que ejercita el conocimiento, y puede ser tanto facultad sensorial como intelectual. El objeto o causa objetiva es la cosa que mueve o a la que tiende el conocimiento, como la piedra o el hombre que se ven. La formal es la misma noticia por virtud de la cual la facultad se vuelve cognoscente, como la visión de la piedra o del hombre. La instrumental es el medio por el cual el objeto es representado a la facultad, como la imagen exterior o estatua de César representa a César. Y, por parte de la causa objetiva o del objeto, debe decirse que el objeto es triple: sólo motivo, sólo terminativo, y a la vez motivo y terminativo. El sólo motivo es el que mueve a la facultad a formar una noticia, no de sí mismo sino de otra cosa, como la imagen o estatua del emperador mueve a conocer al emperador. El sólo terminativo es la cosa conocida por una noticia producida por otro objeto, al modo como el emperador es conocido por su imagen o estatua. El motivo y terminativo a la vez, es el que mueve a la facultad a formar un conocimiento de sí mismo, como cuando se ve la pared en sí misma. ${ }^{15}$

Al igual que Soto, Poinsot nota que hacer conocer es más amplio que representar, y representar más que significar. Pues hacer conocer se dice de todo aquello que concurre al conocimiento, pudiendo decirse de cuatro maneras: efectiva, objetiva, formal e instrumental. De manera efectiva, se dice de la misma facultad que ejercita el conocimiento y de las

13 Cfr. D. de Soto, Op. cit., pp. 3v-4r.

14 Cfr. J. de Sto. Tomás, Op. cit., pars II, q. 21, a. 6, pp. 685b-686a.

15 Cfr. Ibid., pars I, lib. 1, c. 2, p. 9ab. 
otras causas que concurren a él, como Dios que mueve, el intelecto agente que produce las especies, el hábito que ayuda, etcétera. De manera objetiva, se dice de la misma cosa que se conoce. De manera formal, se dice de la misma noticia que, por modo de forma, vuelve cognoscente a la facultad. De manera instrumental se dice del medio que lleva el objeto a la facultad, como la imagen del emperador lleva al emperador al intelecto como un medio, y a este medio lo llamamos instrumento. En cambio, representar se dice de todo aquello por lo que algo se hace presente a la facultad, y se dice de tres maneras: objetiva, formal e instrumental. De manera objetiva se representa el objeto, como la pared. De manera formal, la noticia representa su objeto. $Y$ de manera instrumental lo hace, por ejemplo, la huella. Finalmente, significar se dice de aquello que hace presente algo distinto de sí, por lo que sólo se dice de dos maneras: formal e instrumental. ${ }^{16}$

\section{División del signo}

Hemos visto que, según puntualiza Soto, el representar y el significar son diferentes, pues son como superior e inferior. Esto se ve en que representar se define como "hacer conocer" y significar como "representar". Y, así, el significar puede ser una representación que procede formalmente o una que procede instrumentalmente; es decir, hay signo formal y signo instrumental. Significar formalmente consiste en ser una noticia formal de la facultad, o también consiste en representar por sí y no mediante otro. Significar instrumentalmente se da cuando una cosa, preexistiendo un conocimiento previo de sí misma, representa algo distinto de sí misma. De este modo, para el signo instrumental se requieren dos noticias: (i) la noticia del mismo instrumento - por ejemplo, de la palabra oral o escrita-, y (ii) la noticia de la significación - por ejemplo, de lo que significa esa palabra. ${ }^{17}$

Araújo recoge la misma división, que no postula como pri-

16 Cfr. Ibid., p. 9b. En este sentido, excluye del representar y del significar la causalidad eficiente, cfr. Ibid., pars II, q. 21, a. 5, p. 680 a.

${ }_{17}$ Cfr. D. de Soto, Op. cit., p. 4r. 
mera división del signo, ya que procede en base a la relación entre el signo y la facultad; y más primaria es la relación entre el signo y su designado o significado. En cuanto al signo formal, pone como ejemplo el concepto, que representa su objeto de manera inmediata, sin necesidad de un conocimiento previo del concepto en sí mismo, sino simultáneamente a su conocimiento. Con esto parece postular como signo formal a la especie (inteligible y sensible), con exclusión de otros signos que se consideran como formales, y que son materiales y exteriores, por ejemplo, la estatua. ${ }^{18}$

Poinsot admite también que esta división del signo en formal e instrumental se basa en la relación del signo a la facultad cognoscitiva, y por lo mismo, no es la división primaria del signo. Sus definiciones son las mismas de Soto. Define el signo formal como la noticia formal que representa por sí misma y no mediante otra cosa. El signo instrumental es el que, por un conocimiento preexistente o previo de sí mismo, representa algo distinto de sí mismo, como la huella del buey representa al buey. ${ }^{10} \mathrm{Y}$ dice también que "la división del signo en formal e instrumental es esencial, unívoca y adecuada". ${ }^{20}$

Como siguiente peldaño, Soto pone la otra división que se puede aplicar al signo, a saber, en natural, convencional y consuetudinario.

(i) El signo natural o el significar naturalmente es significar por la naturaleza de la cosa, i.e. sin ninguna imposición ni costumbre. Este modo de significar se subdivide en tres: significar de manera natural común, de manera natural propia y como instrumento natural.

(a) El significar de manera natural común se da cuando la cosa se representa objetivamente a sí misma. Pero este miembro de la división es abusivo, por lo cual debe eliminarse. Sólo deben contarse el significar de manera formal propia y el significar como instrumento natural.

18 Cfr. F. de Araújo, Op. cit., dub. 1, praen. 2.

19 Cfr. J. de Sto. Tomás, $O p$. cit., pars I, lib. 1, c. 2 p. 10a.

20 Ibid., pars II, q. 22, a. 1 , concl. 2, p. 696b. 
(b) El significar de manera natural propia equivale a lo que hemos llamado significar de manera formal, ya explicado. Por lo demás, debería cambiar su nombre por el de "significar de manera natural formal". De este modo la noticia significa en cuanto semejanza natural del objeto. Y también debería cambiarse el nombre de "significar como instrumento natural" por el de "significar como instrumento formal".

(c) Además, el significar como instrumento formal es, en definitiva, significar de manera natural instrumental; es decir, es un signo natural instrumental, como el humo representa al fuego y el gemido la enfermedad, a modo de instrumentos naturales. $\mathrm{Y}$ algunos llaman a esto significar por instinto de la naturaleza. "Pero — puntualiza Soto-, según creo, no cualquier instrumento natural significa por instinto de la naturaleza, sino sólo aquel que significa por institución de la naturaleza, como lo hace la interjección: por ejemplo, el gemido significa el afecto del alma. Sólo para esto hace la naturaleza al gemido, para que exponga y signifique la enfermedad; sin embargo, ni el humo, ni la huella impresa en el polvo han sido producidos para significar, sino que, en cuanto son efectos naturales, les es natural representar a sus causas." ${ }^{21}$ De este modo, el signo natural queda dividido en formal e instrumental.

(ii) El signo convencional o el significar de manera convencional es significar por imposición e institución. Y de esta manera significa no sólo las voces, sino otras cosas, por ejemplo, levantar la mano en clase es signo de pedir la palabra. Y no son signos de esta manera las palabras privadas, sino que tienen que ser públicas. ${ }^{21}$ Estas últimas -las palabras públicas- son palabras y lenguaje por "imposición auténtica". El significar de manera convencional tiene dos modos: de manera formal y consecutiva o por translación. Un ejemplo de la manera formal y primigenia se da cuando se pone nombre a un niño. Esto constituye una significación

21 Cfr. E. Villanueva, El argumento del lenguaje privado, México: UNAM, 1979 , p. 14. 
propia. Un ejemplo de la manera consecutiva y por translación o transferencia se da cuando un nombre se lleva o se transfiere a significar otra cosa por semejanza o por algún otro sentido figurado, como llamar "Nerón" a un hombre cruel, por semejanza con ese emperador; o decir "se esconde la culebra en la hierba", en sentido de que se esconde un fraude bajo la apariencia de humildad. Esto constituye una significación impropia.

(iii) El signo consuetudinario o el significar por costumbre es significar por un cierto uso sin imposición. Por ejem. plo, el perro que siempre va delante de su amo, lo significa; o como el mantel sobre la mesa, que antecede a la comida, y la significa. Pero esto no se puede llamar "institución" o "imposición", como lo hacen algunos, sino que es más bien algo natural. Por eso, esta clase de signos puede reducirse a la de los signos naturales instrumentales. Así, pues, de alguna manera Soto se inclina a reducir la división del signo sólo en natural y convencional. ${ }^{22}$

Araújo hace eco al intento sugerido por Soto de reducir esta división del signo a signo natural y signo artificial o convencional. También se dice que es la división primera del signo, dado que la relación primaria del signo es hacia lo designado, y no tanto hacia la facultad. ${ }^{23}$ En este sentido, critica la división de Fonseca ${ }^{24}$ en natural y convencional; subdividido este último en convencional por imposición y convencional por costumbre. Araújo no la acepta, porque "por imposición" y "por convención" son lo mismo. Por eso prefiere la de Soto, quien divide primariamente el signo en natural y convencional $\mathrm{y}$, como subdivisiones del natural, el perfecto y el imperfecto o consuetudinario. Así, al ser imperfecto, no tiene significación real, sino de razón, teniendo fundamento en la costumbre, que imita a la naturaleza. ${ }^{25}$ Pero queda convenientemente ubicado dentro de los signos naturales.

22 Cfr. D. de Soto, Op. cit., p. $4 \mathrm{v}$.

23 Cfr. F. de Araújo, Ibidem.

24 Cfr. P. de Fonseca, Dialectica, lib. 1, c. 9.

25 Cfr. F. de Araújo, Op. cit., dub. $i$, resp. ad. obiect. 
Por su parte, Poinsot plantea esta adecuada división del signo simplemente como una división trimembre por razón de la relación entre el signo y lo designado: natural, convencional y consuetudinario. El signo natural es el que representa por la naturaleza de la cosa, sin ninguna imposición ni costumbre; y así representa lo mismo para todos, como el humo representa al fuego. El signo convencional es el que representa algo por imposición de la voluntad a través de la autoridad pública, como la voz "hombre". El signo consuetudinario es el que representa sólo por el uso, sin imposición pública, al modo como el mantel sobre la mesa significa la comida. ${ }^{26}$ Por lo demás, dice que esta división puede tomarse, desde diferentes puntos de vista, como análoga o como unívoca: "Si esta división del signo en natural y convencional se considera entitativamente y según el ser de la cosa, es análoga; y, si se toma en el género de lo representativo o cognoscible, es unívoca." 27

\section{Ontología del signo}

La perspectiva más importante es, sin duda, la ontológica o metafísica. En ella es decisiva la labor de Araújo, que Poinsot sabrá formular de manera magistral. Ambos consideran que lo propiamente ontológico del signo, más que la sola materialidad del mismo signo, que por sí sola no agota la riqueza de la significación, es la relación de significación, $\mathrm{y}$, por tanto, el signo, ontológicamente considerado, pertenece a la categoría de la relación. Su contribución a la semiótica universal en este punto es admirable.

\section{El signo en cuanto tal}

El constitutivo formal del signo, para Araújo, es la relación (sin establecer aún qué tipo de relación, lo cual dependerá de los distintos tipos de signo). Pero, aunque es una relación simple, tiene dos aspectos, direcciones o términos: a lo de-

${ }^{26}$ Cfr. J. de Sto. Tomás, $O p$. cit, pars I, c. 2, p. 10a.

27 Ibid., pars II, q. 22, a. 5, concl. unic., p. $715 \mathrm{a}$. 
signado y a la facultad. Por eso Araújo tiene que esclarecer el estatuto de cada uno de esos términos y cuál es el principal. Para hacerlo, procede primero negativamente, diciendo lo que no es tal relación. En primer lugar, niega que la relación del signo sea sólo entre éste y lo designado: "El signo no se constituye por el solo orden a lo designado con exclusión de la facultad." ${ }^{28}$ La prueba de esto se toma óptimamente de la definición misma del signo, que también incluye la relación a la facultad, y, por lo tanto, tal relación pertenece a su razón formal. En segundo lugar, niega que la relación a lo designado y a la facultad sean iguales: "El signo no mira a la facultad y a lo designado por igual, ni el orden a la facultad es un modo completivo del orden a lo designado." ${ }^{29}$ La razón de esto es que la facultad y lo designado no pueden integrar el término adecuado de una relación simple como es la que constituye al signo. Y es que, con el mismo orden esencial con el que dice relación a lo designado, también dice relación a la facultad, pues ambos son aspectos esenciales suyos. Por tanto, es ilusorio poner, como lo hacen algunos, que la relación a la facultad es un modo completivo de la relación a lo designado. Con esto puede ya establecer la solución positiva: "El signo se constituye esencialmente por una única y simple relación terminada primariamente en lo designado y secundariamente en la facultad." de probar mejor este aserto lo divide en tres partes. Prueba que tiene como término primario de su relación a lo designado: "Aquello por lo que se define un relativo y con lo que se dice convertiblemente es su término primario; pero el signo se define por orden a lo designado y se dice convertiblemente con él, pues el signo es signo de lo designado y es representativo de él, como se dice en su definición; luego ..." ${ }^{31}$ Aduce otro argumento para probar que la facultad cognoscitiva es el término secundario de su relación: "Cuan-

\footnotetext{
28 F. de Araújo, Op. cit., dub. 2, concl. 1.

29 Ibid., concl. 2

30 Ibid., concl. 3.

31 Ibid., prob. 1.
} 
do un ente respectivo mira a dos términos con cierto orden, mira a uno ut quod y al otro ut cui, y al uno primariamente y al otro secundariamente ... ; luego, de manera semejante, ya que el signo mira a lo designado como algo que debe volver o representar a la facultad, mira a aquél de modo primario y a éste de modo secundario." ${ }^{32} \mathrm{Y}$ que se refiere a ambos términos con una sola relación simple, lo prueba así: "Cuando dos términos son respectivos con cierto orden, uno ut quod y otro ut cui, éstos no bastan para duplicar la relación, sino que terminan exactamente la misma porque no son términos totales, y que la terminan por igual. ... Luego el signo, por la misma relación, mira a lo designado como representable a la facultad." "ss Más aún, puede decirse que la relación del signo tiene dos efectos, uno esencial y otro accidental. El efecto esencial es referirse a lo designado; el accidental es conducir a la facultad al conocimiento de lo designado y representado; este efecto accidental es separable, pues proviene del fundamento de la relación del signo como de la razón formal y de la misma relación del signo como de cierta condición concomitante. Así, aun cuando la relación a la facultad es importante, sin embargo, más importante es la relación a lo designado.

Así, pues, el constitutivó formal del signo es visto en la tradición tomista como una relación. Lo propio del signo es decir relación a lo designado y a la facultad cognoscitiva. Por eso Poinsot, siguiendo la línea de Araújo, establece que la razón formal del signo es la relación. Por eso remite al tratado de la relación, como un requisitivo previo para comprender la formalidad ontológica del signo. ${ }^{* 4}$ De acuerdo con ello, dice: "La razón del signo, formalmente hablando, no

32 Ibid., prob. 2.

33 Ibid., prob. 3.

s4 Esto lo señala muy bien J. N. Deely, "The Two Approaches to Language: Philosophical and Historical Reflections on the Point of Departure of Jean Poinsot's Semiotic", en The Thomist, 38 (1974), p. 873. Para una exposición detallada de la teoría tomista de las relaciones, cfr. A. Krempel, La doctrine de la relation chez Saint Thomas, Paris: Vrin, 1952. 
consiste en la relación según el decir, sino según el ser." \$s Resalta, entonces, la fuerza ontológica que se da al significar, como una relación. De modo que el signo no se reduce a ser una entidad material o mental tomada de modo absoluto, sino relativa a algo - a lo designado y a la facultad-y en esta relación tiene precisamente su ser de signo, de otra manera quedaría reducido al ser de objeto. Al igual que Araújo, Poinsot se percata de que el signo indica relación a dos cosas: a lo designado y a la facultad. Por eso se pregunta si mira a ellos con una sola relación, o están ahí invisceradas varias relaciones. También como Araújo, resuelve que se trata de una sola relación con dos términos desigualmente alcanzados y, cuando se trata propiamente al signo, se trata de una sola relación simple: "Si la facultad y lo designado se consideran como términos directamente alcanzados por la relación, necesariamente exigen dos relaciones en el signo, pero de este modo el signo mira a la facultad directamente como objeto, no formalmente como signo. En cambio, si se considera la facultad como término alcanzado en oblicuo, así, con una única relación del signo se alcanzan lo designado y la facultad, y ésta es la razón propia y formal del signo."

35 J. de Sto. Tomás, Op. cit., pars II, q. 21, a. 1, concl unic., p. 647a. Allí mismo trats de las relaciones, pars II, q. 17, pp. 573b-608b. Por ser muy útil, damos el resumen que hace Josef Gredt: "La relación, tomada en sentido muy amplio, es el orden u ordenación de una cosa a otra. Esto orden puedo estar incluido en alguna esencia absoluta [no en el sentido de 'divina', sino en el de 'no-relativa'] o ser un respecto puramente adventicio o sobreañadido a la esencia absoluta. La ordenación incluida en una esenoia absoluta es la relación secundum dici. Esta es en verdad un ente absoluto pero que connota esencialmente algo extrínseco, en orden al cual exige ser definido; asf, la relación del alma con el cuerpo es una relación secudum dici. En cambio, el orden adventicio, que consiste en un puro respecto, o aquel orden cuyo ser completo es haberse a otro, es la relación secundum esse; por ejemplo, la relación de paternidad, que es un puro respecto adventicio al hombre. La relación secundum esse puede ser real y de rapón. Es real la que tiene ser en la naturaleza de las cosas, independientemente de la consideración de la mente, como la relación de padre a hijo; es de razón la que sólo subsiate en el intelecto, como la relación de predicado a sujeto" (I. Gredt. Elementa philosophiae aristotelica-thomisticae, Friburgo-Barcelona: Herder, 1956 (1la. ed.), vol. I, p. 154).

s6 J. de Sto. Tomás, $O p$. cit., pars II, q. 21, a. 3, concl. unic., p. 664ab. 


\section{El signo natural}

El constitutivo formal del signo es ser una relación. Tanto Araújo como Poinsot establecen que es una relación según el ser; pero en cuanto abarca a la relación real y a la relación de razón. En este sentido, Araújo se pregunta si la relación del signo es real en todos los signos o de razón en todos y, si no se da un solo tipo de relación en todos, en cuáles la relación es real y en cuáles es de razón. Establece que en los signos naturales es real, no de razón. ${ }^{37}$ Como prueba principal puede aducirse el siguiente argumento: es relación formalmente real la que tiene una causa real de su ser y que compete al sujeto por fuerza de su producción real; pero la relación del signo natural es de esta manera; luego es real $^{38}$ También Poinsot trata en un artículo el problema de si en el signo natural la relación es real o de razón. Y responde en el mismo sentido que Araújo: "La relación del signo natural a su designado, por la que se constituye en el ser de signo, es real, y no de razón, en cuanto está de su parte y por fuerza de su fundamento, y suponiendo la existencia del término y las demás condiciones de la relación real." " ${ }^{\prime a} \mathrm{Y}$ estas condiciones de la relación real ya habían sido tomadas en cuenta por Araújo, en especial la existencia del término o extremo significado. Por ejemplo, para que una huella signifique al animal designado, debe existir el prototipo que es el animal. Cuando este prototipo no existe, por ejemplo, en el caso de la huella de un buey, que por su naturaleza ha sido instituida para que signifique a ese animal, pero éste ha muerto, la relación ya no puede ser real -falta una condición requerida para que lo sea-, y entonces es de razón, pero por aceidente, y sólo a causa de ese defecto o falta de una condición requerida. ${ }^{* 0}$ Se vuelve de razón no por la fuerza intrínseca de su ser, sino por la fuerza extrínseca y accidental de una carencia: "Cuando alguna relación por su naturaleza y

\footnotetext{
a7 F. de Araújo, Op. cit., dub. 1, concl. 1.

38 Cfr. Ibid., prob. 1.

39 J. de Sto. Tomás, $O p$. cit., pars II, q. 21, a. 2, concl. unic., p. 656b.

40 F. de Araújo, Op. cit., dub. 1, concl. 2.
} 
por los méritos propios de su sujeto y fundamento debe ser real, pero por un defecto extrínseco no es real, no es real por accidente; pero la relación del signo natural, en cuanto está en su naturaleza y por los méritos de su fundamento, debe ser real ... ; luego cuando falta la existencia del término extrínseco, por accidente falta el ser real, y en lugar de él surge otra relación de razón." "11

\section{El signo convencional}

En cuanto a los signos convencionales, sin embargo, Araújo establece que se constituyen por una relación de razón, la cual es un ente de razón de primera intención. ${ }^{22}$ Que sean relaciones de razón, lo prueba así: "Hay una relación de razón cuando no se ordena algo por su propia naturaleza, sino por una comparación de la razón que compara a una cosa con otra; pero los signos convencionales no se ordenan por la naturaleza de las cosas a sus designados, sino por la ordenación extrínseca del intelecto que los instituye para significar; luego ..."43 Que sean de primera intención lo prueba así: "La intención primera ... es aquella que inmediatamente sigue a una acción real, y en ella se funda; pero de este modo es la relación del signo [convencional], ya que, puesta la institución activa, en seguida surge la significación de las voces, $y$, en otras cosas libremente instituidas para significar, en seguida el intelecto les puede atribuir la relación de signo y usarlas para significar; luego ..."44 No otra cosa resuelve Poinsot, quien dice: "En los signos convencionales la razón de signo también debe explicarse por su relación a lo desig. nado. Pero esta relación es de razón, y el signo no consiste sólo en una denominación extrínseca por la que es impuesto o destinado por la república para significar." 45

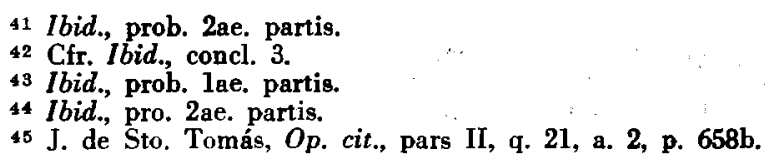




\section{El signo formal}

Ya que los signos formales son naturales, la relación por la que se constituyen es una relación real. El signo formal es aquel en el cual y por el cual se conoce lo designado, es decir, contiene en si mismo lo significado y, en cuanto objeto, este signo en cierta manera se oculta para sólo dejar resplandecer lo representado. El ejemplo por excelencia de esto es el concepto, el cual es un signo que representa en sí mismo y por sí mismo lo significado. Tanto Araújo como Poinsot lo aceptan. El primero lo establece así: "La razón de signo natural [y formal] se encuentra univocamente y con toda propiedad en los conceptos de nuestro intelecto." $\mathrm{La}$ razón es que el concepto o palabra interior es causa de la palabra exterior, y lo que es causa de que otro signifique es signo de manera más propia que su efecto significante. Poinsot precisa aún más: "El concepto es especie expresa del intelecto y de manera muy propia es signo formal." ${ }^{47}$ En las especies cognoscitivas, ya intelectuales, ya sensoriales, podemos distinguir entre especie expresa, que es la más perfecta, y especie impresa. Hemos visto que la especie expresa intelectual es aceptada como signo formal por Araújo y Poinsot. Ambos aceptan además que la especie expresa sensible es signo formal.

Araújo aclara que es menos propia que la inteligible, pero que es signo formal: "En las noticias sensitivas también se encuentra verdadera razón de signo formal, aunque no de manera tan perfecta como en los conceptos." ${ }^{48}$ La razón es que las especies expresas sensibles son, al igual que las inteligibles, formalmente representativas del objeto en sí mismas y por sí mismas. Lo mismo dice Poinsot: "También la imagen (idolum) o especie expresa sensible en las facultades interiores es signo formal respecto de tales facultades." ${ }^{49}$

Pero excluyen ambos las especies impresas, tanto inteligi.

46 F. de Araújo, Op. cit., dub. 3, concl. 1.

17 J. de Sto. Tomás, Op. cit., pars II, q. 22, a. 2, p. 704a.

18 F. de Araújo, Op. cit., dub. 3, concl. 3.

$49 \mathrm{~J}$. de Sto. Tomág, Ibid. 
bles como sensibles. Araújo lo formula así: "La especie impresa, ya sensible, ya inteligible, de ninguna manera es signo." "Las excluye en base al siguiente argumento: "De la razón de signo, además del representar algo distinto de sí mismo, se requiere que medie entre el objeto representado y la facultad a la que hace la representación; pero la especie impresa no media así, porque se da por parte de la facultad, como un principio de su mismo conocimiento, de modo que por él la facultad se vuelva facultad cognoscitiva íntegra, que pueda conocer de manera próxima; luego la especie impresa no es signo formal." ${ }^{51}$ El asunto es claro; ya que la especie impresa no es signo, tampoco puede ser signo formal. Por eso Poinsot dice, lacónicamente: "La especie impresa no es signo formal." " Y añade, además, una conclusión semejante sobre el acto de conocer tomado en cuanto tal, por la misma razón que en la especie impresa, a saber, no es representación de lo conocido, sino operación productora de esa representación; así, "el acto de entender, como distinto de la especie impresa y de la expresa, no es signo formal, en cualquier operación del intelecto en que se tome".

\section{El signo instrumental}

El signo instrumental es una relación; si se trata de un signo instrumental natural, dicha relación será real, y, si se trata de un signo instrumental convencional, dicha relación será de razón. Araújo lo distingue del signo formal - y en ello cifra la menor perfección del signo instrumental - en el conocimiento preexistente que requiere para hacer llegar a la facultad al conocimiento del objeto designado como con cierto discurso. ${ }^{54}$ Pero hay tres clases de conocimiento previo por

so F. de Araújo, Op. cit., dub. 3, concl. 2.

s1 Ibid, prob.

62 J. de Sto. Tomás, Op. cit., pars II, q. 22, a. 3, concl. unic., p. 708a.

53 Ibid., a. 4, concl. unic., p. 712b. Cfr. J. A. Casaubón, "Para una teoría del signo y del concepto mental como signo formal", en Sapientia, 10 (1955), p. 272; y J. G. Pola, "Noción tomista del "signo formal", en Studium, 5 (1965), p. 98.

o4 Cfr. F. de Araújo, Op. cit., dub. 4, concl. unic. 
las cuales se puede tocar al signo instrumental, por ejemplo, una estatua: (i) De un primer modo, ese conocimiento previo puede terminarse en la estatua en cuanto es una cosa que tiene cierta figura y líneas. (ii) De un segundo modo, puede terminarse en la estatua en cuanto es un signo, i.e. en cuanto se refiere al prototipo al que indica relación, de modo que se conozca su relación de signo en acto signado o como cierta quididad, por conocer la estatua como algo representativo. (iii) De un tercer modo, puede terminarse en la estatua en cuanto subyace a la relación de signo, no como cierta quididad y en acto primero, sino en cuanto ejerce un segundo signo que es la actual representación de lo designado y la conducción de la facultad al conocimiento de éste. Según esto, Araújo resuelve: "El conocimiento previo del signo instrumental no es del tercer modo, sino del primero y sobre todo del segundo, pues para el conocimiento de la cosa significada se requiere previamente la noticia del instrumento significante, a saber, qué cosa es y además que tiene tal significación." Y Y no se puede confundir el signo instrumental con el objeto sólo motivo; porque el signo tiene relación primaria a lo designado y secundaria a la facultad, diciéndose convertiblemente del designado; mientras que el objeto tiene relación primaria con la facultad y secundaria con lo conocido, diciéndose convertiblemente con la facultad; pues el objeto motivo se dice tal por la facultad, en tanto que el sig. no instrumental se dice tal por lo designado.

\section{El signo consuetudinario}

Para establecer el tipo de relación que ejerce el signo consuetudinario, hay que saber primero si es signo natural o convencional. Si lo primero, tendrá relación real; si lo segundo, tendrá relación de razón. Poinsot se encarga de resolver este problema, diciendo que a veces el signo consuetudinario puede ser convencional y a veces natural: "Si la costumbre se refiere a algún signo, destinándolo y proponién-

55 Ibidem. 
dolo como signo, tal signo fundado en la costumbre será convencional. Pero si la costumbre no propone o instituye algo como signo, sino que indica el simple uso de alguna cosa y por razón de él se toma como signo, tal signo se reduce al natural." ${ }^{58}$ En algunos casos, pues, el signo consuetudinario es convencional, y entonces ejerce una relación de razón; en otros casos, en cambio, es signo natural, aunque imperfecto, y entonces ejerce una relación real, como compete a los signos naturales. Un ejemplo de lo primero es la institución de algunas voces especiales para designar ciertas cosas por costumbre; un ejemplo de lo segundo es el dar al mantel sobre la mesa el significado de la comida próxima; pero esto último es más cercano al signo natural, ya que se funda en la repetición y el hábito, que es como una "segunda naturaleza."

\section{Psicología del signo}

En la tradición tomista el estudio psicológico del signo se encuentre entreverado con las consideraciones lógicas y metafísicas. El origen y el funcionamiento del signo como fenómeno psicológico humano era tratado junto con los aspectos lógicos de definición y división del mismo, y junto con sus fundamentos metafísicos. Pero hay en esta tradición un gran cuidado en establecer su génesis y funcionamiento en las distintas facultades psíquicas. El signo se gesta ya desde los sentidos, que producen especies sensibles propias, como sentidos externos. Estas especies sensibles propias se reducen a una especie sensible común por el sentido interno o "sensus communis" que les da estructura. Como especie sensible expresa, ya se constituye en signo primordial, que gana en significatividad en cuanto pasa a la imaginación. Después, la "vis cogitativa" da a la imagen su estatuto más propio de signo, pues la imaginación ve la cosa en la imagen, pero la cogitativa ve la imagen como imagen o signo de la cosa. $Y$, por último, la intervención del intelecto es la decisiva, pues

5e J. de Sto. Tomás, Op. cit., pars II, q. 22, a. 6, p. 719a. 
con ella el signo adquiere su estatuto completo de signo, además de que la especie expresa intelectual es también un signo. De este modo, por el intelecto los signos se convierten plenamente en signos. $\mathrm{Y}$, por otra parte, los signos del intelecto, los conceptos, son - respecto del lenguaje- "palabras interiores", que fundamentan y causan la significación de las "palabras exteriores", que son las expresiones linguiísticas como tales. ${ }^{57}$ Además, la memoria opera con los signos tanto como memoria sensitiva cuanto como memoria intelectiva. $Y$ se considera el funcionamiento del signo en relación con la afectividad.

\section{Gnoseologia del signo}

Igualmente importantes son las relaciones del conocimiento con el signo, sea con el signo en cuanto objeto, sea con el signo en cuanto que establece una ordenación a lo significado. En cuanto a este punto, son notables los estudios de Poinsot sobre los vehículos del conocimiento, las "notitiae", a las que dedica una cuestión después de las dos propias del signo. ${ }^{58}$ Lo mismo respecto de los diferentes objetos, en relación con la cognoscibilidad del signo y su relación con la facultad cognoscitiva, que marcan la pauta gnoseológica del funcionamiento del signo como elemento cognoscible y especialmente como elemento que hace conocer. ${ }^{88}$

\section{Conclusión}

Por muchos motivos merece ser conocida, revitalizada y aplicada la semiótica tomista. Con los complementos que le brinda la semiótica actual, puede convertirse en una gran ayuda para la comprensión filosófica del fenómeno semiótico.

s7 Cfr. F. Soria, "Filosofía del signo", en Estudios Filosóficos, 12 (1963), especialmente el núm. 2: "Estudio psicológico del signo", pp. 416 ss. Cfr. además R. Verneaux, "La communication par signes", en Saint Thomas d'Aquin aujourd'hui, Recherches de Philosophie, VI, Paris: Desclée de Brouwer, 1963, pp. $79-80$.

ss Cfr. J. de Sto. Tomás, Op. cit., pars II, q. 23: De notitiis et conceptibus, pp. $722 \mathrm{a} 8 \mathrm{~s}$.

so Cfr. Ibid., pars II, q. 21, a. 4, pp. 670a ss. 
This article attempts to make a synthesis on the classic Thomist reflection on the sign. Our study focuses on what is known as "the Thomist Renaissance" of the 16th and 17th centuries. In our opinion this is best represented by Domingo de Soto, Francisco de Araújo, and Juan de Santo Tomás née Juan Poinsot. Because of both the chronology and school affiliations, we are presenting the authors and their respective problems in order of succession and importance. Although explicit references are not numerous, we can perceive, respectively, Poinsot's debt to the doctrine of Araújo and Araujo's to that of Soto. Soto was the great logician and theorist of the sign; Araújo, the conscien. tious analyst and critic; and Poinsot, the excellent systematizer of problems, polemics, and conclusions. The famous adage circulating in the Spanish universities of the time (especially in Salamanca) referred to Soto: "qui scit Sotum, scit totum"; Araújo called him "magister noster", and Poinsot quoted him with great esteem. The sequence among these thinkers is therefore apparent.

The reflection on the sign encompassed various fields: ontology, logic, psychology, and epistemology; but here we will be primarily concerned with the ontological and logical aspects.

The relational character of the ontology of the sign must be insisted upon, much as Peirce has done a long time afterwards. The sign maintains a relationship with the thing signified and with the cog. nitive faculty. Numerous problems arise in specifying the type of relationship the sign has with each of these correlates. The three thinkers struggle to explain the ontological relationship implied by the sign.

The classification of the various types of signs, then, is very interesting. It is extensive and complicated, but it does show us our thinkers' lucidity with respect to the multiple modalities of signification.

The properties of each sign, distinct and characteristic, were also examined in terms of their own nature, since the different nature of each type of sign makes for different properties. The authors considered the many difficulties inherent in the division of signs and in differentiating among them according to their respective properties.

As to the logic of the sign, we find that these philosophers did a study that we, today, would not hesitate to call "semiotic". They explain and ponder the functions of the sign as the basis for all 
instruments of logic: in the order of terms, in the order of propositions, and in the order of inferences. It should be noted that they paid careful attention to syntax, semantics, and pragmatics.

We find important observations - although still in incipient and rudimentary form - on the pragmatic aspect of the sign. They also offer us a great wealth of syntactical and semantic considerations. Above all, in the semantics sector, a strong realism is evident, easily on a par with contemporary developments. In addition, there are some psychological and epistemological theories on the sign that these authors work with.

In conclusion, recent research on the subject of the comparison between scholastic logic and contemporary logic has demonstrated how little we know about the former and the many lessons it could teach the latter, since it elaborates a profoundly philosophical reflection on the phenomenon of the sign.

[M. B.] 